\title{
Numerical study of the low-Reynolds flows in the vertical heated caverns
}

\author{
Mikhail Petrichenko ${ }^{1}$, Vitaly Sergeev ${ }^{1}$, Darya Nemova $^{1 *}$, Evgeny Kotov ${ }^{1}$, and Darya Tarasova ${ }^{1}$ \\ ${ }^{1}$ Peter the Great Saint-Petersburg Polytechnic University, 195251 Polytechnicheskaya, 29, St.-Petersburg, Russia
}

\begin{abstract}
The object of research is the critical geometry of a three-dimensional air flow in a cavern between two vertical heated plates. In this rate the convection's contribution to heat transfer will be limited due to thermal conductivity at a fixture temperature drop. A three-dimensional RANS approach closed by the k-w SST turbulence model in conjunction with the energy equation. The model validated and verified by comparison with the experimental results. The results of the work applied in developing of ventilated façades.
\end{abstract}

\section{Introduction}

The thermal insulation of the building used in hinged ventilated facade is one of the main factors promoting decrease the thermal losses. However, the insulation eventually collapses under the influence of various phenomena, loses its integrity, structure and thermal insulation properties. In addition, thermal insulation materials for facade walls are quite expensive. In addition, the energy efficiency of the building can be improved using non-conventional energy sources [1].

One of the possibilities to exclude the use of thermal insulation materials in ventilated facade systems is the use of closed caverns in the air gap, which the geometric dimensions allows to minimize the contribution of air convection to heat transfer. Natural convection in between two vertical plates has been studied by many researchers under different types of boundary conditions on the channel walls in the presence or absence of channel's input and output effects. The main attention in the literature is paid to the case of lifting flow at symmetric heating of vertical plates.

Wright J. L., Jin H., Hollands K. G. T., Naylor D. experimentally studied the natural convection of gas (air) at $\operatorname{Pr}=0.7$ in a vertical cavity with various heat aluminum walls. The flow was visualized using smoke and a laser light source [2].

In [3-6], considerable attention is paid to the stability of stationary convective flows. The authors have made many studies of flows between flat parallel walls. To simulate the flow and heat transfer of a liquid in a vertical channel, unsteady two-dimensional NavierStokes equations in the vorticity-current function variables were solved. There is a graph of the dependence of the minimum critical Grasgof's number on the Prandtl number when the flow instability is observed. In [7], the authors studied the linear stability of the natural convection of a liquid between vertical plates of different temperatures using the collocation method. It was found that for $\operatorname{Pr}<12.45$ the critical Grasgof's number is almost independent of the Prandtl number (for $\operatorname{Pr}=0.71$ and $\operatorname{Pr}=7$ the field stability differs little, while for $\operatorname{Pr}>12.45$ the instability threshold depends noticeably on the Prandtl number).

The analysis of heat transfer performance of nanofluids for laminar flow was fully made with forced convection with two zones: one adiabatic and one with uniform wall heat flux. In particular, the heat transfer coefficient of water-based nanofluids is increased by 3.4$27.8 \%$ under fixed Reynolds number compared with that of pure water. Also, the enhancement of heat transfer coefficient is larger than that of the effective thermal conductivity at the same volume concentration [8-10].

A lot of research about convective heat transfer were conducted numerically [11-25].

However, to date, the prospects for the use of threedimensional caverns in the air gap of ventilated facade systems have not been determined.

The purpose of this paper is determination the critical geometric dimensions of a three-dimensional vertical heated cavern. In this rate, the flow in the threedimensional cavern will be close to the stationary and convection's contribution to heat transfer will be limited due to thermal conductivity at a fixture temperature drop. The results of the work can be applied by the development of ventilated facade systems. To achieve this goal, it is necessary to solve the following tasks:

1. To develop a mathematical model of a threedimensional RANS approach closed with the k-w SST turbulence model in conjunction with the energy equation

2. To perform numerical simulation in the ANSYS software package to determine the characteristics of the heat flow, and also for the validation and verification of the model by comparison with the experimental results

\footnotetext{
* Corresponding author: darya0690@mail.ru
} 


\section{Materials and methods}

\subsection{Mathematical model of flow in vertical heated cavern}

To consider the air flow a rectangular area elongated in the vertical direction is selected (Fig.1). The ratio of height to width is varying in the range $A>20$. On the side walls the constant temperature condition is set, the upper and lower walls are adiabatic.

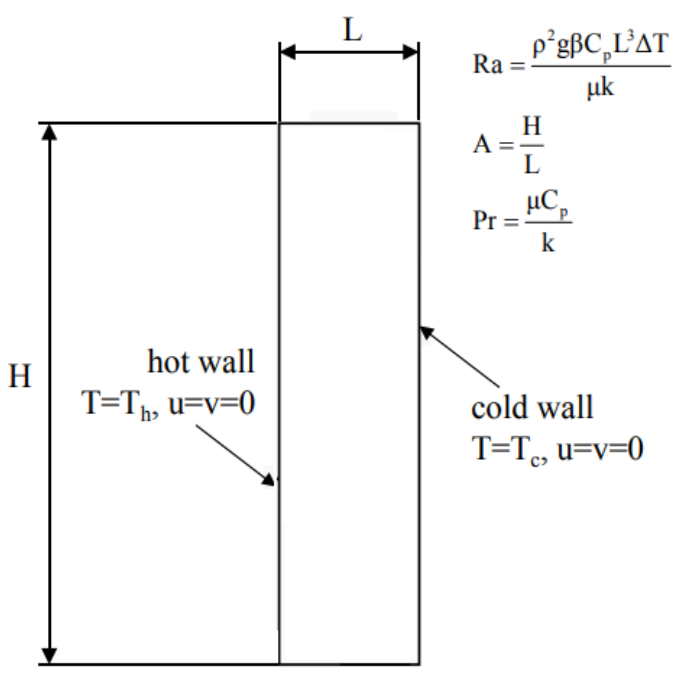

Fig. 1. Computational domain for two-dimensional formulation

Convection and heat transfer in the presented calculation described by Navier-Stokes equations in their nonstationary formulation taking into account the Bossiness' approximation. In this case it reduces the problem to the calculation of incompressible fluid and gas flow in the presence of mass force proportional to the local temperature drop. We obtain the equations:

$\nabla \cdot V=0$

$\partial V / \partial+(V \nabla) V=-(1 / \rho) \nabla \cdot p+v \nabla \cdot V+g \beta T \gamma$

$\partial T / \partial+v \nabla T=a \nabla T$

$V$ - velocity of the liquid;

$T$ - temperature;

$p$ - modified pressure;

$\rho$ - average density;

$g$ - acceleration of gravity;

$v$ - coefficient of kinematic viscosity,

$a$ - coefficient of thermal conductivity;

$\beta$ - coefficient of volumetric expansion;

$\gamma$ - unit vector directed vertically upwards.

Introducing dimensionless variables: distance - $h$, time$h^{2} / v$, velocity - g $\beta \Delta T h^{2} / v$, temperature - $\Delta T$ (temperature difference), pressure - $p \mathrm{~g} \beta \Delta T h$, we obtain a system:

$$
\nabla \cdot V=0
$$

$\partial V / \partial t+G r\left[(\mathrm{~V} \nabla) V_{o}+\left(V_{o} \nabla\right) V\right]=-\nabla p+W+T_{\gamma}$

$\partial T / \partial t+G r\left[\mathrm{~V} \nabla \mathrm{T}_{0}+\mathrm{V}_{0} \nabla \mathrm{T}\right]=1 / \mathrm{P}_{\mathrm{r}} \nabla \mathrm{T}$
The velocity and temperature profiles of the main current in dimensionless variables $V_{o}$ and $T_{o}$ have the form:

$V_{o}=1 / 6(x-x), T_{o}=-x$

The problem contains two dimensionless parameters that determine the similarity of convective flows-the Grasgof's and Prandtl number:

$\operatorname{Pr}=v / a, G r=\left(\mathrm{g} \beta \Delta T L^{3}\right) / v^{2}$

\subsection{Numerical simulation of flow in vertical heated cavern}

For the calculations, an area having a size of $\mathrm{L} \times 20 \mathrm{~L} \times$ 20L was chosen. The problem was solved in a dimensionless setting. Initially the calculations were performed in three-dimensional formulation for Rayleigh numbers $R a=7300$ and $R a=15000$. Calculations for Rayleigh numbers $R a=7300$ are performed in a nonstationary laminar setting. Temperature fields in different sections of the computational domain are shown in figure 2 .

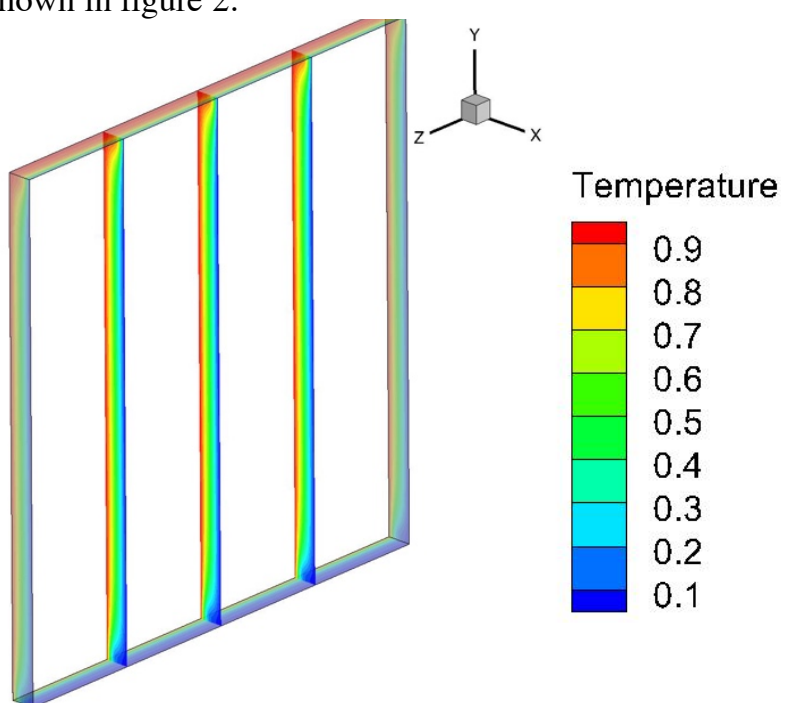

Fig. 2. Temperature fields in different sections of the computational domain for Rayleigh numbers $\mathrm{Ra}=7300$.

The three-dimensional calculation is performed to show that at Rayleigh number $R_{a}=7300$ the flow inside the cavern is stable in the third direction. This is evident from the temperature profiles in different sections. Similar results were obtained for the Rayleigh number $R_{a}=10000$. The current became unsteady, but was not changed in any section of XY. From this, we can conclude that for the calculation of flows with lower Rayleigh number we can limit ourselves to a twodimensional formulation.

\section{Results and discussions}

The results of calculations are presented in the central vertical section of the calculated area for all the Rayleigh numbers considered, since the size of the calculated area in the third direction does not affect the obtained results (Fig. 3-4). 
Since at Rayleigh numbers below $R_{a}=10000$ the flow is stable in the third direction, it is possible to proceed to the two-dimensional formulation.

Figure 3 shows the longitudinal velocity fields depending on the Rayleigh number.

With an increase of the Rayleigh number, the flow becomes nonstationary but it remains two-dimensional.

The flow is two-dimensional and laminar for all considered flow regimes. Calculations for Rayleigh numbers $R_{a}=6800$ and $R_{a}=7300$ showed convergence only in non-stationary formulation.
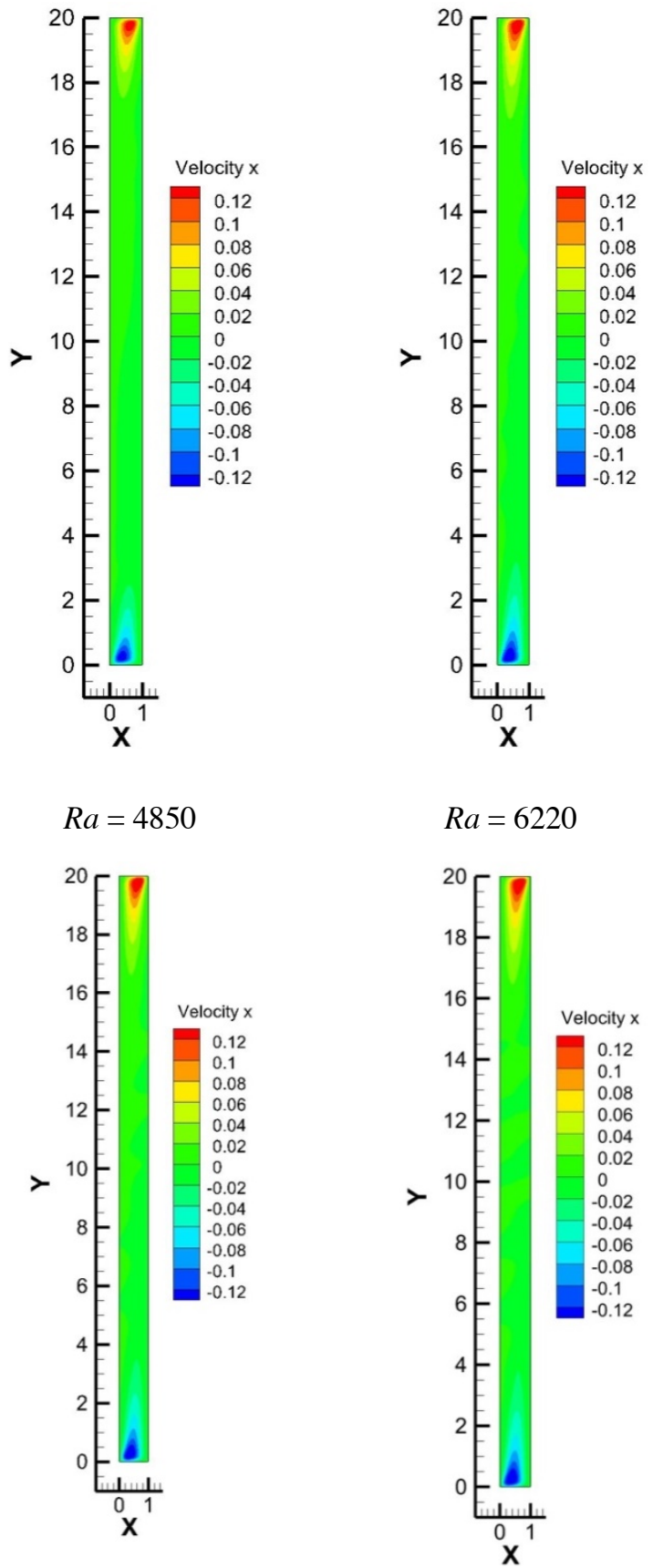

$$
R a=6800
$$$$
R a=7300
$$

Fig. 3. Fields of longitudinal velocity in the vertical crosssection of the calculated area for different Rayleigh numbers.
The temperature fields Rayleigh numbers less than $R_{a}$ $=10000$ are qualitatively similar. The temperature field for $R_{a}=4850$ are shown in figure 4 .

It assumes that the heat transfer resistance of the cavity can be calculated as:

$$
R=\delta / \lambda
$$

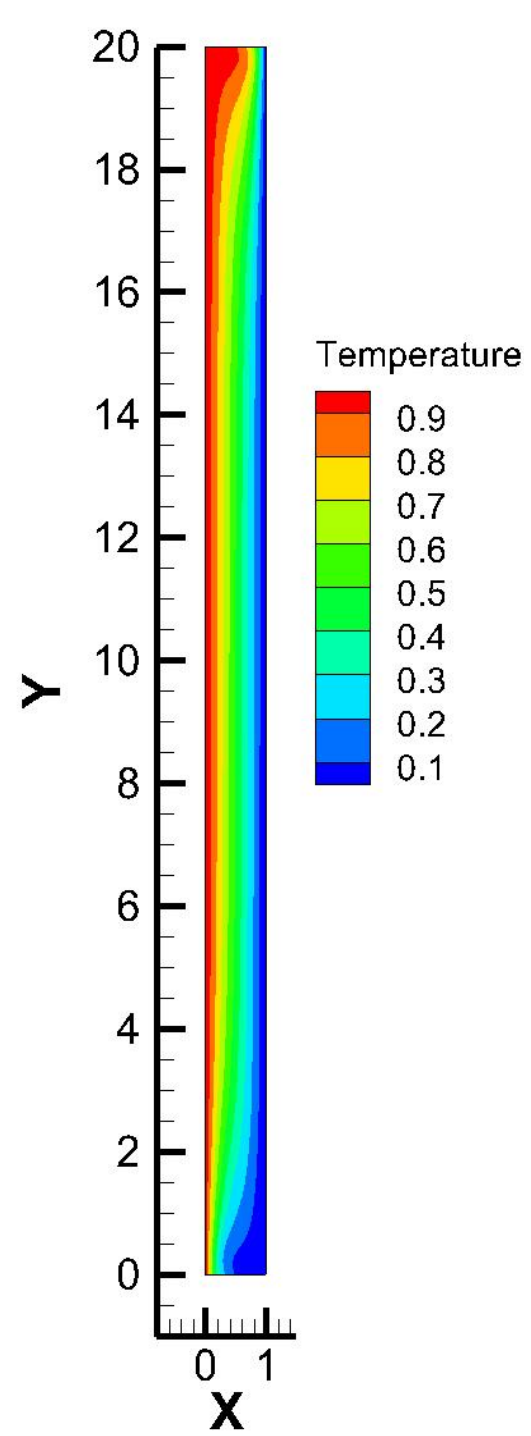

Fig. 4. The temperature field for $R a=4850$.

Since the critical Rayleigh number is known, at which there is no transition to a three-dimensional flow, it is possible to estimate the temperature difference on the walls that permissible for the operation of the cavern as a heat insulator:

$$
\Delta T \leq 10000\left(v a / g \beta L^{3}\right)
$$

As the number $\mathrm{Ra}$ increases, the linearity gradually disappears. The linearity limit is marked at $\mathrm{R}_{\mathrm{a}}=10000$. If condition (5) is performed, the temperature distribution across the cavern is linear and the thermal resistance of the cavern is determined only by its thickness and the coefficient of molecular thermal 
conductivity of the air. Linear temperature profiles are shown at Fig.5.

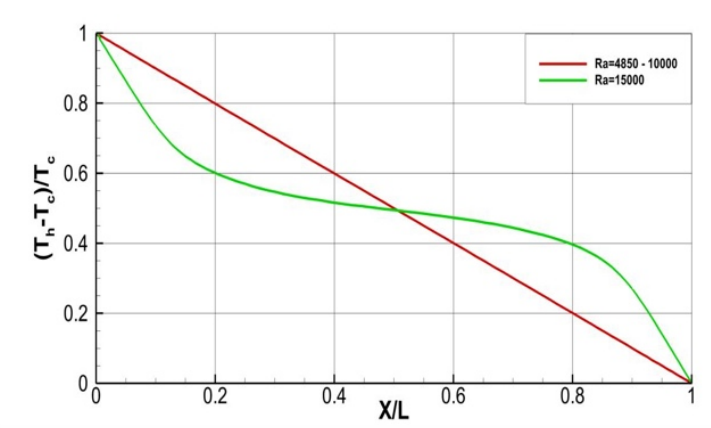

Fig. 5. The linear temperature profiles

If condition (5) is achieved, the cavern with air consider as a heat-conducting solid medium. It is possible to achieve the equivalence of its thermal properties to the insulation properties by varying the geometric dimensions of the cavern. Thus, these results can be applied in the design of facade systems by using a vertical cavern with air instead of insulation.

\section{Conclusion}

It can be concluded from the obtained results:

1. A stable non-stationary flow regime is observed for such Rayleigh number, because the temperature fields in different cross-sections of the flow coincide.

For the flow with Rayleigh number $R_{a}=15000$, the nonstationary formulation without the turbulence model did not give the required convergence on residuals. So it was calculated by using the RANS approach closed by the k-w SST turbulence model. In this case the flow is unstable in the third direction, therefore it is impossible to consider the cavern as a heat insulator at numbers $R_{a}=10000$ and above.

2. A long narrow channel, viscous free-convective flowover approximated by two-dimensional over.

3. The stability of the flow in vertical cavern with symmetric conditions is proved.

4. The occurrence of the circulation flow is due to the asymmetry of the limiting temperature conditions at the ends of the cavern.

5. Thus, in conditions of low-Reynolds viscous flow, the cavern plays the role of a thermal insulator with thermal resistance $h / \lambda$, where $\lambda$ is the coefficient of thermal conductivity of air.

6. If condition $\Delta T \leq 10000\left(\mathrm{va} / \mathrm{g} \beta L^{3}\right)$ is performed, the temperature distribution in the cavern is linear and the thermal resistance of the cavern is determined only by its thickness and the coefficient of molecular thermal conductivity of the air.

7. If condition $\Delta T \leq 10000\left(\mathrm{va} / \mathrm{g} \beta L^{3}\right)$ is achieved, it is possible to consider the cavern with air as a heatconducting solid medium. By varying the geometric dimensions of the cavern, it is possible to achieve the equivalence of its thermal properties to the insulation properties. Thus, these results can be applied in the design of facade systems by using the vertical cavern with air instead of insulation.

\section{References}

1. C. López-Ordóñez, J. Roset, G. Rojas-Cortorreal, Archit. City Environ., 12 (34) (2017)

2. J. Wright, H. Jin, K. Hollands, D. Naylor, Int. J. Heat and Mass Transfer, 49 (2006)

3. D. Hopper, D. Jaganathan, J. Orr, J. Shi, F. Simeski, M. Yin, J.Liu, Nanofluids, 7 (6) (2018)

4. W. Lewandowski, M. Ryms, , Denda, H. Int. J. Therm. Sci. 134 (2018)

5. S. Amala, B. Mahanthesh, Nanofluids, 7 (6) (2018)

6. M. Leporini, F. Corvaro, B. Marchetti, F. Polonara, M. Benucci, Exp. Therm. Fluid Sci., 99 (2018)

7. G. McBain, S. Armfield, Int. ANZIAM J., 45 (2004)

8. A. Minea, S. Murshed, Renewable Sustainable Energy Rev., 91 (2018)

9. E. Chereches, I., K. Sharma, V., A. Minea, Continuum Mech. Thermodyn., 30 (3) (2018)

10. A. Minea, W. El-Maghlany, Eng. Sci. 174 (2017)

11. A. Gorshkov, N. Vatin, P. Rymkevich, O. Kydrevich, Mag. Civ. Eng. , 78 (2) (2018)

12. M. Ananin, N. Perfilyeva, I. Vedishcheva, N. Vatin, IOP Conf. Ser. Mater. Sci. Eng., 365 (2) (2018)

13. A. Chechevichkin, N. Vatin, V. Samonin, M. Grekov, Mag. Civ. Eng., 76 (8) (2017)

14. E. Khrapunov, Y. Chumakov, Phys. Conf. Ser., 1038 (1) (2018)

15. N. Yaroslavtseva, N. Ivanov, Phys. Conf. Ser. 929 (1) (2017)

16. E. Khrapunov, I. Potechin, Y. Chumakov, S. Phys. Conf. Ser. 891 (1) (2017)

17. N. Kharkov, Phys. Conf. Ser. 891 (1) (2017)

18. E. Vitokhin, E. Ivanova, Continuum Mech. Thermodyn., 29 (6) (2017)

19. Y. Luo, J. Zhang, W. Li, E. Sokolova,Y. Li, W. Minkowycz, Numer Heat Transfer Part A Appl., 71 (12) (2017)

20. E. Statsenko, T. Musorina, A. Ostrovaia, V. Olshevskiy, A. Antuskov, Mag. Civ. Eng., 70 (2) (2017)

21. E. Loktionova, D. Malyshevsky, D. Schemelinin, D. Zaborova, Adv. Trends Eng. Sci. Tech. Proc. Int. Conf. Eng. Sci. Tech., pp. 531-536 (2017)

22. T. Musorina, V. Olshevskyi, A. Ostrovaia, E. Statsenko, MATEC Web Conf., 73 (2016)

23. J. Sorokins, A. Borodinecs, J. Zemitis, IOP Conf. Ser. Earth Environ. Sci., 90 (1) (2017)

24. P. Ovchinnikov, A. Borodinecs, K. Strelets, Build. Environ., 112 (2017)

25. D. Baranova, D. Sovetnikov, D. Semashkina, A. Borodinecs, Procedia Eng. 205 (2017). 